\title{
EVALUATION OF THE COMPRESSIVE RESPONSE OF NOTCHED COMPOSITE PANELS USING A FULL-FIELD DISPLACEMENT MEASUREMENT SYSTEM
}

David M. McGowan and Damodar R. Ambur

NASA Langley Research Center

Hampton, VA 23681-0001

and

T. Glen Hanna and Stephen R. McNeill

University of South Carolina

Columbia, SC 29208

Presented at the 40th AIAA/ASME/ASCE/AHS/ASC Structures,

Structural Dynamics and Materials Conference

Session No. 39 - Analysis of Composite Structures III

Paper No. 99-1406

St. Louis, Missouri

April 12-15, 1999 


\title{
EVALUATION OF THE COMPRESSIVE RESPONSE OF NOTCHED COMPOSITE PANELS USING A FULL-FIELD DISPLACEMENT MEASUREMENT SYSTEM
}

\author{
David M. McGowan* and Damodar R. Ambur \\ NASA Langley Research Center \\ Hampton, VA 23681-0001 \\ and \\ T. Glen Hanna ${ }^{\ddagger}$ and Stephen R. McNeill ${ }^{\S}$ \\ University of South Carolina \\ Columbia, SC 29208
}

\begin{abstract}
$\underline{\text { Abstract }}$
An experimental and analytical evaluation of the compressive response of two composite, notched stiffened panels representative of primary composite wing structure is presented. A three-dimensional full-field image correlation technique is used to measure all three displacement components over global and local areas of the test panels. Point-wise and full-field results obtained using the image correlation technique are presented and compared to experimental results and analytical results obtained using nonlinear finite element analysis. Both global and global-local image correlation results are presented and discussed. Results of a simple calibration test of this image correlation technique are also presented.
\end{abstract}

\section{Introduction}

One of the requirements for airframe structures development is the demonstration of structural damage tolerance for a panel with a two-stringer-bay-wide notch. The response of notched panels made of composite materials and loaded in compression or tension is not well understood. Experimentally validated analysis methods that predict the damage initiation and growth at the notch location are needed. Such methods can eventually be used to predict the residual strength of composite primary structures. Evaluating the response of structures with discontinuities such as cutouts and damage in the form of notches requires full-field information that represents the experimental in-plane and out-of-plane displacement components of the structure. Such information is also needed to investigate and understand the coupling effects in anisotropic plates and geometrically nonlinear deformation effects in thin structures.

The full-field measurement techniques that provide accurate data are currently limited to conventional interferometric methods such as moiré techniques and laser interferometry. ${ }^{1}$ The moiré techniques require positioning a grid with fine lines very close to the test speci-

\footnotetext{
${ }^{*}$ Aerospace Engineer, Structural Mechanics Branch. Senior Member, AIAA.

${ }^{\dagger}$ Assistant Head, Structural Mechanics Branch. Associate Fellow, AIAA.

${ }^{\ddagger}$ Research Assistant, Dept. of Mechanical Engineering.

${ }^{\S}$ Associate Professor, Dept. of Mechanical Engineering.
}

men. Therefore, the use of these techniques can potentially interfere with the deformed specimen. Furthermore, only one displacement component can be measured with a given grid and equipment configuration. These disadvantages make moiré techniques impractical for simultaneously measuring all of the displacement components that are large in magnitude. Laser interferometric techniques require a controlled environment with an elaborate and cumbersome setup for measuring all displacement components.

Davidson $^{2}$ has demonstrated a Scanning Electron Microscope based technique that provides accurate inplane displacements around stiffness discontinuities. This method, however, can only monitor small regions of the test article. Another more promising method is the three-dimensional image correlation technique described in Ref. 3. This field-displacement measurement technique utilizes a camera-based stereo vision system to monitor the three-dimensional locations of points on the surface of the loaded test specimen. This system is a non-intrusive system since the only part of the measurement system that comes into contact with the test specimen is the speckle pattern that is applied to the surface of the specimen to establish the displacement tracking points. The resolution of the measurement data is a function of the speckle pattern density and the distance between the cameras and the specimen. The accuracy of the displacements measured in the present study range from $\pm 3.5 \times 10^{-5}$ in. to $\pm 1.7 \times 10^{-3}$ in.

Copyright (C) 1999 by the American Institute of Aeronautics and Astronautics, INC. No copyright is asserted in the United States under Title 17, U.S. Code. The U.S. Government has a royalty-free license to exercise all rights under the copyright claimed herein for government purposes. All other rights are reserved by the copyright owner. 
Another application of this displacement measurement technique is to monitor displacements in a global-local manner. In such an application, coarser full-field measurements are made over a large area of the specimen, and much finer measurements are made over a smaller, local area within the larger area. The local area monitored may be centered about some structural feature or damage. These types of measurements can be very important to obtaining a better understanding of the effects of local details like cutouts or damaged areas on the load distribution in built-up structures.

The present paper describes an experimental and analytical evaluation of the compressive response of two composite stiffened panels with a central notch. Point-wise and full-field results obtained using the three-dimensional image correlation technique mentioned previously are presented and compared to experimental results obtained using conventional displacement measurement devices and analytical results obtained using nonlinear finite element analysis. The threedimensional image correlation procedure and a simple calibration test are also described.

\section{Image Correlation Procedure and Calibration Test}

The theoretical development for stereo imaging is well documented. ${ }^{3-9}$ The main difficulty in making accurate three-dimensional measurements lies in the application of the underlying theory. In fact, threedimensional measurements in experimental mechanics using computer vision concepts are relatively recent, ${ }^{6-8}$ where Luo et al. used an image correlation algorithm that matched square subsets in one image to square subsets in another and then used these data to triangulate and estimate the three-dimensional displacement field. The lack of perspective correction in the matching process limits the range of camera orientations that can be used for accurate image evaluation. The projection/back-projection method developed recently by Helm et al., ${ }^{3}$ known as VIC3D (Video Image Correlation in 3 Dimensions), overcomes these limitations and extends the use of the measuring system to a greater range of problems. Furthermore, because the measurement procedure uses features on the surface of the object rather than a projected pattern, the system is capable of true, point-to-point, surface displacement measurements for both curved and flat panels.

The VIC3D system developed for this work has two main parts. The first part is used for the calibration of the camera system, and the second part uses a digital image correlation procedure to determine the full, threedimensional surface displacement field. A schematic of the measurement system is presented in Fig. 1. Brief descriptions of both the calibration and measurement components are presented subsequently.

\section{VIC3D System Calibration}

Calibration of the two-camera computer vision system, VIC3D, determines the relative positions and operating characteristics of both cameras. The calibration system is based on a series of images of a grid with a known line spacing. Each camera is

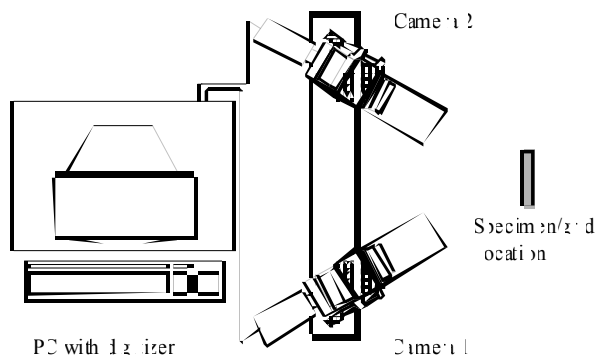

Fig. 1. Schematic of the three-dimensional displacement measurement system.

calibrated to the grid individually but, because the position of each camera is known relative to the same grid, their relative position to each other is also known.

The camera and lens system is modeled as a pinhole device. To increase the accuracy of the model, it is modified to correct for Seidel lens distortion. ${ }^{9}$ The pinhole camera projection equations that govern the use of these cameras have been described in previous publications. ${ }^{4,5}$ The imaging characteristics of a camera modeled in this manner can be described by five parameters. The pinhole distance (phd) is the perpendicular distance from the pinhole to the sensor plane of the camera and can be considered the magnification factor of the lens. The location of the center of the image $\left(C_{x}, C_{y}\right)$ is defined as the point on the sensor whose normal passes through the pinhole. These parameters are important because lens distortion is proportional to the cube of the distance from the center of the image. The lens distortion factor, $\kappa$, is a correction for Seidel lens distortion. The final parameter is the aspect ratio, $\lambda$, of the sensors. The value of $\lambda$ is the ratio of the size of a pixel in the $\mathrm{Y}$ direction to its size in the $\mathrm{X}$ direction.

Six parameters $X_{0}, Y_{o}, Z_{0}, \alpha, \beta$ and $\gamma$ are required to describe the relationship between a camera and the coordinate system of the calibration grid. ${ }^{3}$ The parameters $X_{0}, Y_{o}$ and $Z_{o}$ describe the position in space of the grid relative to the camera, and $\alpha, \beta$ and $\gamma$ describe the angular orientation of the grid relative to the camera. 
To calibrate a camera, an image of the grid is taken. The camera is then moved a known distance perpendicular to its sensor plane and a second image is taken. Using (a) the known spacing of the grid, (b) the known movement of the camera, and (c) the location of the grid intersections, as extracted from the calibration images, nonlinear optimization can be used to find values for the parameters that best describe the position and operating characteristics of the camera. The process is then repeated for the second camera without moving the grid.

\section{VIC3D Measurements}

Once calibration is completed, the VIC3D stereo vision system can be used to measure object shape (profile measurements) and full-field, three-dimensional displacements. The VIC3D system uses a random speckle pattern that is applied to the test specimen surface to provide a unique set of features to map from one camera to the other.

Profile Measurements. The projection method used for profile measurements is shown schematically in Fig. 2. The analysis uses a pair of images taken of the surface of the object by camera 1 and camera 2 at the same point in time. A small square section of the image taken by camera 1 is projected onto the candidate plane in space. Assuming that the surface of an object can be modeled using a series of small planar patches, each candidate plane is described relative to camera 1 by two of the plane's direction angles $\theta, \varphi$ and the variable $\mathrm{Z}_{\mathrm{p}}$ denoting the location of the intersection of the plane and the optic axis for camera $1 .^{3}$

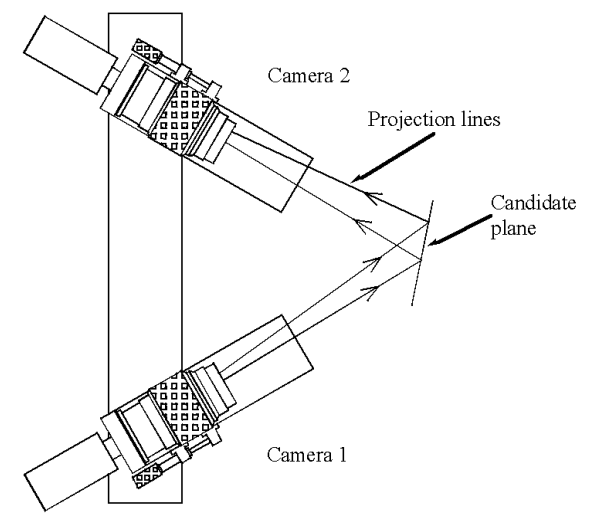

Fig. 2. Projection method used for profile measurements.

The initial position of the candidate plane is established through an interactive first guess generator. Because the operating characteristics of camera 1 are known, the gray levels contained in the image of the subset can be projected onto the candidate plane to create a virtual gray level pattern in space. The virtual gray level pattern is then projected into camera 2. This process creates a second virtual gray level pattern at the sensor of camera 2. A cross-correlation error function between the recorded gray level pattern from camera 2 and the virtual gray level pattern projected onto the sensor of the second camera is used to obtain optimal estimates for the candidate plane variables. Once the system has been optimized, the three dimensional position of that point on the surface of the object can be calculated from the camera 1 parameters and the position of the candidate plane.

Displacement Measurements. The displacement measurement system requires that a set of images be taken before the object is loaded (undeformed images) and a second set be taken after the load has been applied to the specimen (deformed images). The first part of the displacement measurement system is the same as that of the profile measurement system. An initial candidate plane is chosen and a virtual gray level pattern in space is established. As with the profile system, the virtual pattern is projected back into camera 2. At this point, the virtual gray level pattern in space is allowed to translate and rotate in a rigid-body manner to a second position, as shown in Fig. 3.

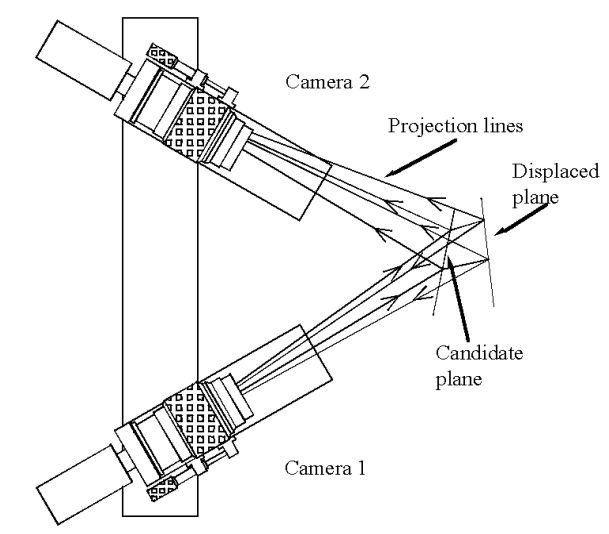

Fig. 3 Projections used for the displacement measurement system.

The displaced virtual gray level pattern is then projected back to the sensor planes in cameras 1 and 2. As with the profile system, a cross-correlation error function is established between the virtual gray level patterns at the sensor planes of cameras 1 and 2 and the recorded gray level patterns from the deformed images recorded by those cameras. The three error functions are simultaneously optimized establishing both the position and displacement of that point on the surface of the object. By continuing the analysis of other portions of the undeformed camera 1 image, the profile and three- 
dimensional displacement of the surface of the object is measured for the entire field of view shared by both cameras.

\section{Calibration Test}

A simple tension test of a flat 2024-T3 aluminum specimen with a centered, machined notch was used to calibrate the VIC3D system with more conventional displacement measuring devices currently being used at the NASA Langley Research Center Structures and Materials Laboratory. A schematic of the calibration test specimen is shown in Fig. 4(a). The specimen is 12-in. long by 3.5 -in. wide and 1/16-in. thick.

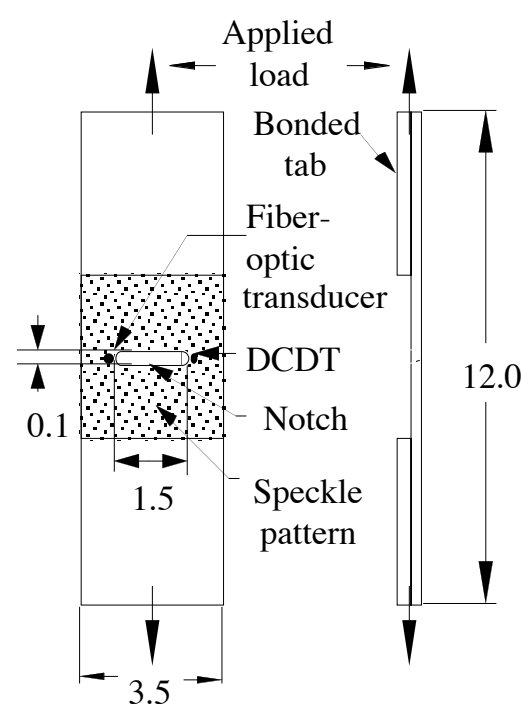

(a) Schematic of the notched aluminum test specimen

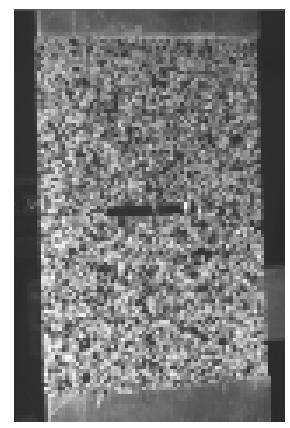

(b) Speckle pattern

Fig. 4. Details of the aluminum calibration specimen (dimensions are in inches).

The specimen also has 1/16-in.-thick tabs bonded to one side only to induce additional out-of-plane displacements due to bending. The notch is 1.5-in. long by $0.1-$ in. wide. The specimen was loaded in tension by a hydraulic test machine with a 50-kip maximum load capacity.

The speckle pattern shown in Fig. 4(b) was applied to the specimen by spray painting. A thin layer of white epoxy paint was sprayed onto the surface. Epoxy paint was used to insure that the speckle pattern deformed with the surface, as well as to avoid cracking that may occur with other paints. Shortly after applying the epoxy paint, the speckles were applied with a black enamel spray paint. The black paint spray can nozzle was altered in order to create a splattering effect, as opposed to a fine mist. The paint was then allowed to dry for 24 hours. This method enabled a highcontrast, random speckle pattern to be produced, as shown in Fig. 4(b). The details of the VIC3D setup used for this test are given in Ref. 10, and they are not repeated here. The full-field displacement measurements were determined to be accurate for this test to within $\pm 3.5 \times 10^{-5}$ in.

Point-wise out-of-plane displacements were measured using a direct current displacement transducer (DCDT) at one notch tip and a fiber-optic displacement transducer at the other notch tip. Both of these devices were located on the same surface of the specimen. The VIC3D displacement measurement system was used to monitor full-field displacements over a 4-in.-long by 3.5-in.-wide area on the surface of the specimen opposite to these two devices (see Fig. 4(a)). The ends of the specimen were supported by hydraulic grips in the test machine, and the specimen was loaded in tension to a load of approximately 4,500 lbs.

Results from the full-field measurements indicate that some misalignment of the specimen was present when the specimen was mounted into the test machine, causing a slight twisting of the specimen. This twist in the specimen is indicated by the profile of the specimen that was calculated using the VIC3D system. A three-dimensional view of the initially twisted specimen is shown in Fig. 5.

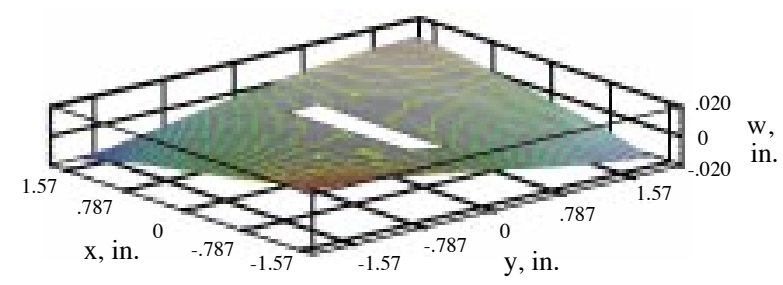

Fig. 5. Initial profile measurement of the aluminum calibration specimen.

The ability to measure initial profiles of test specimens is an important feature of the VIC3D system.

A comparison of results obtained from the three displacement measurement techniques is presented in 
Fig. 6. The results from the present full-field displacement measurement system are compared to the pointwise results by displaying the displacements of the "speckle" that is most nearly opposite to the location of each of the point-wise devices.

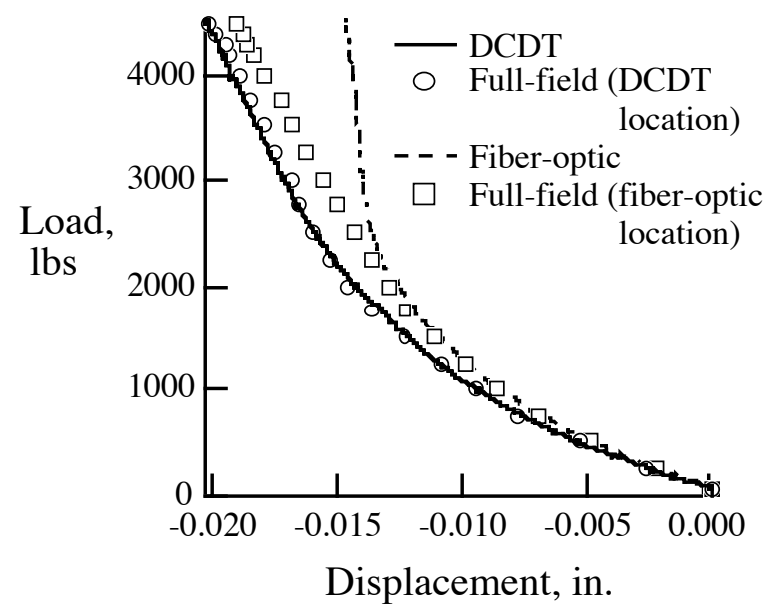

Fig. 6. Comparison of DCDT, fiber optic, and fullfield system displacement results for the tension calibration specimen.

The point-wise results are plotted in Fig. 6 as curves, and the full-field results are plotted as symbols. As shown in the figure, the agreement between the DCDT measurements and the full-field measurements is very good. The agreement between the fiber optic measurements and the full-field measurements is very good for applied loads less than approximately 2,500 lbs. The disagreement between the results for loads greater than 2,500 kips is attributed to the fact that, during loading of the specimen, the fiber optic device exceeded the calibration range for which it was set up to measure displacements. This result is evidenced by the fairly flat displacement profile measured with this device for applied loads greater than 2,500 lbs.

\section{Test Panels, Apparatus, and Test Conditions}

The two composite panels studied in the present paper are stiffened panels that were machined from either the upper or lower cover panels of a 12-foot-long, stitched graphite-epoxy wing box manufactured by the Boeing Company (formerly McDonnell Douglas) as part of the NASA Advanced Composites Technology (ACT) program. Although a complete description of this wing box is given in Ref. 11, a brief description of the materials used to fabricate the two test panels is given here. The cover panels were fabricated from Hercules, Inc. AS4/3501-6 and IM7/3501-6 graphite-epoxy materials that were stitched together using E.I. DuPont de Nemours, Inc. Kevlar ${ }^{\circledR}$ thread. IM7 graphite fibers were only used as the 0-degree fibers in the lower cover panel. The skin and stringers of the panels were fabricated from prekitted stacks of nine layers of dry graphite material forms. Each prekitted stack of material was approximately 0.055 inches thick and had a [45/$\left.45 / 0_{2} / 90 / 0_{2} /-45 / 45\right]_{\mathrm{T}}$ laminate stacking sequence. The skin and stringers of the wing box were fabricated by stitching several of these prekitted stacks of material together to provide the desired thickness at different locations in the wing box. The wing box was fabricated using the Resin Film Infusion (RFI) process described in Ref. 12 .

A schematic of the panels studied in the present paper is given in Fig. 7. As shown in the figure, these three-stringer panels had a 7-in.-long by 3/16-in.-wide notch machined through the center stringer.

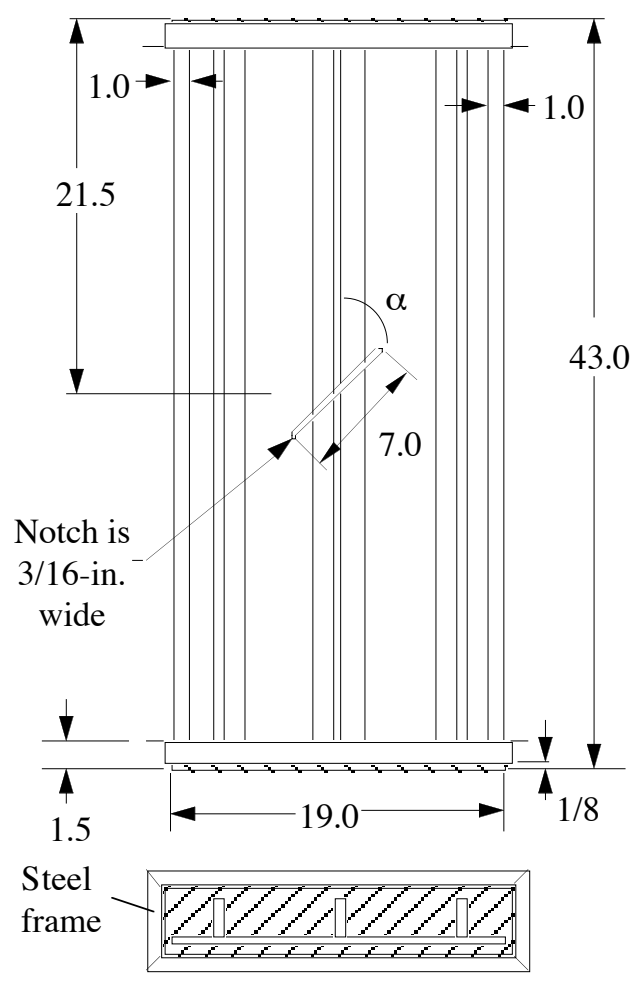

Fig. 7. Schematic of a stitched, graphite-epoxy panel with a centered, machined notch through the center stringer (dimensions are in inches).

The notch in each panel was oriented at an angle $\alpha$ to the center stringer. The value for $\alpha$ for the upper cover panel was $90^{\circ}$ and the value of $\alpha$ for the lower cover panel was $60^{\circ}$. The height of the stringer blades was $2.38 \mathrm{in}$. for the upper cover panel and $2.0 \mathrm{in}$. for the lower cover panel. The loaded ends of both panels were 
encased in 1.5 inches of epoxy potting compound to prevent an end-brooming failure, and those edges were machined flat and parallel to each other. The unloaded edges of the panels were supported with knife-edge supports to provide a simple support boundary condition along those edges.

A photograph of the test set-up for the upper cover panel is given in Fig. 8(a). Full-field displacements were monitored in a region measuring approximately 6.89-in. wide by 4.53 -in. long that was centered horizontally at one of the notch tips.

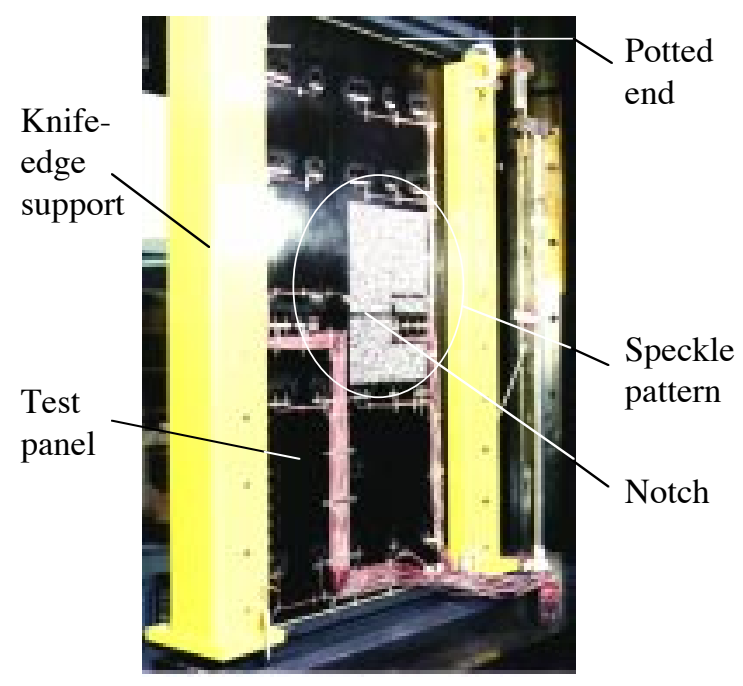

(a) Test setup

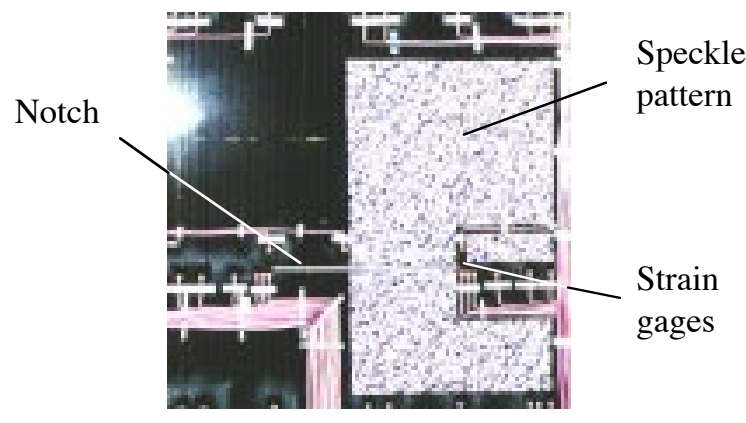

(b) Speckle pattern used for the three-dimensional, fullfield displacement measurement system

Fig. 8. Photographs of the notched composite upper cover panel.

The speckle patterns for the tests of the composite panels were produced by printing a computer-generated pattern on an adhesive-backed Controltac ${ }^{\mathrm{TM}}$ Plus vinyl sheet manufactured by the $3 \mathrm{M}$ company. Details of the use of this material are given in Ref. 10. The speckle patterns were applied to the unstiffened surface of the panels. A close-up view of the speckle pattern used for the test of the upper cover panel is shown in Fig. 8(b). This speckle pattern was discontinuous because the electrical resistance strain gages, identified in Fig. 8(b), that had been installed to monitor the strain field around the notch tip obstructed the view of that area.

A photograph of the lower cover panel is shown in Fig. 9. The global-local three-dimensional image correlation technique was used for this test. The global region monitored measured approximately 15 -in. wide by 27.5-in. long. The local region monitored was approximately 7.7-in. wide by 4.6-in. long, and was centered on one of the notch tips. The speckle pattern used for this test is shown in Fig. 9. The details of the VIC3D setups used for these tests are given in Ref. 10.

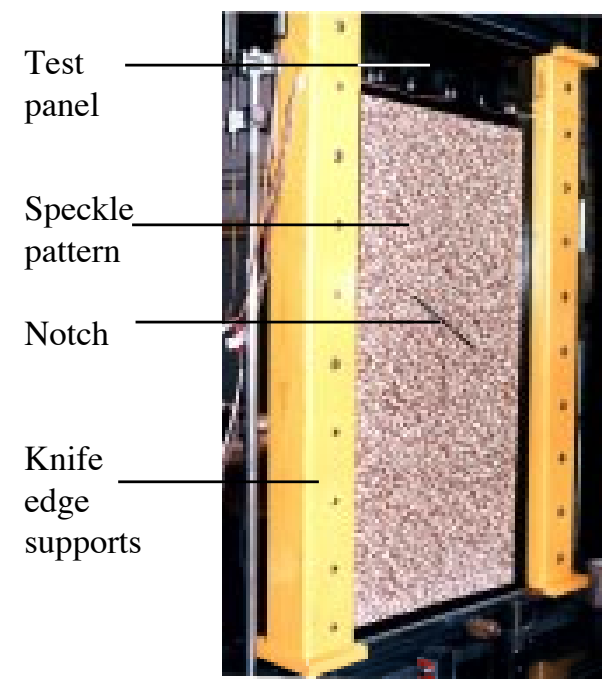

Fig. 9. Photograph of the notched composite lower cover panel.

\section{Analytical Models}

The analytical results described in the present paper were obtained from nonlinear analyses performed using the Structural Analysis of General Shell Structures (STAGS) finite element code. ${ }^{13}$ The finite element model used to analyze the lower cover panel (with $\alpha=$ $60^{\circ}$ ) is shown in Fig. 10(a). A close-up view of this model around the notch is shown in Fig. 10(b). The finite element mesh is much more refined in this region to allow for an accurate representation of the strain field in the vicinity of the notch tip. A similar model was used to analyze the upper cover panel. In both models, the stringer flanges were modeled separately from the skin and eccentricities were used to account properly for the change in thickness in those regions. The nominal elastic material properties used in the analyses are given in Table 1 for the stitched/RFI AS4/3501-6 and 
AS4/IM7/3501-6 material systems used to fabricate the present panels.

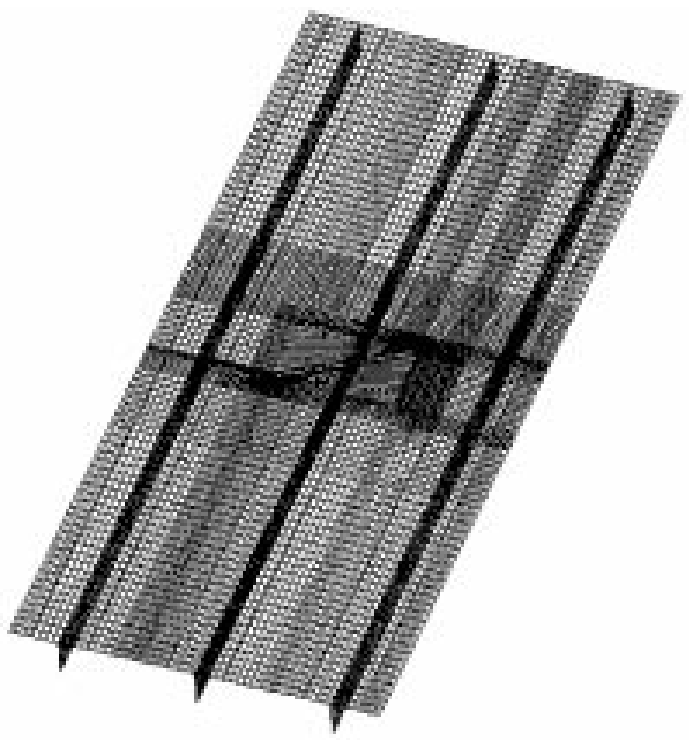

(a) Full model

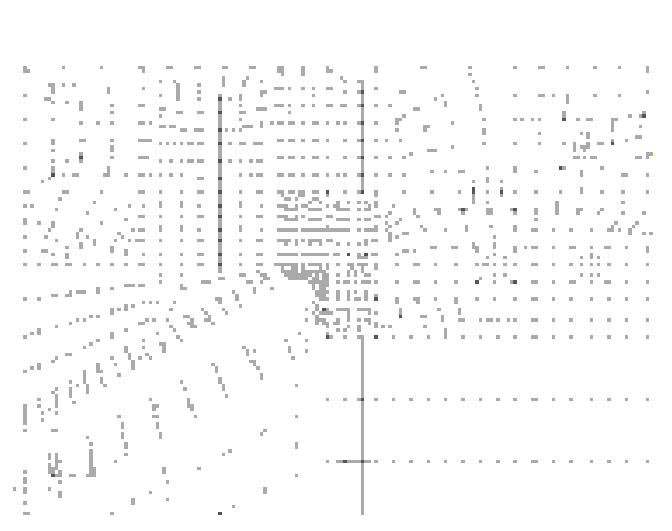

(b) Close up of mesh at notch tip

Fig. 10 Finite element model of the upper cover panel.

\section{Results and Discussion}

Results from the compression tests of the notched upper and lower cover panels are described in this section. Results obtained using the VIC3D image correlation system are presented and compared to the experimental and analytical results. Both point-wise and fullfield results are presented and compared.

\section{Upper Cover Panel Results}

As shown in Fig. 8(b), full-field displacements were measured in a local region measuring approximately 6.89 -in. wide by 4.53 -in. long centered horizontally at one of the notch tips. The full-field results were determined to be accurate for this test to within $\pm 1.4 \mathrm{x}$
$10^{-4}$ in. ${ }^{10}$ A comparison of measurements obtained from a single DCDT located on the stiffener side of the panel and full-field measurements obtained from the skin-side of the panel is shown in Fig. 11. The DCDT was located 1.8 inches above the horizontal centerline of the panel, and it was centered on the blade of the cut, center stringer. Once again, the full-field results are compared to the point-wise DCDT results by displaying the displacements of the "speckle" that is most nearly opposite to that of the DCDT.

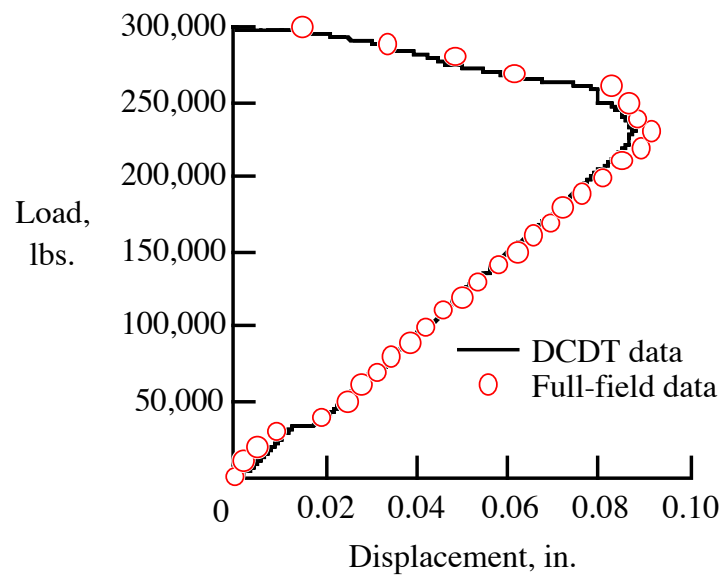

Fig. 11. Comparison of DCDT and full-field displacement results for the notched composite upper cover panel.

The DCDT results are plotted in Fig. 11 as a solid curve, and the full-field results are plotted as symbols. As shown in this figure, the agreement between the full-field results and the DCDT results is very good. The reversal in the direction of the out-of-plane displacement for loads greater than 225 kips was represented very well. A slight separation between the two sets of results for loads greater than 250 kips occurs because the full-field results are obtained for a fixed point on the front surface of the panel, while the DCDT results are obtained for a fixed point in space that corresponds to the DCDT location. Therefore, as the panel shortens under the applied compressive load, the DCDT measures the displacement of several different points on the back surface of the panel as they pass in front of the DCDT.

Results obtained from a geometrically nonlinear finite element analysis of this panel are shown in Fig. 12 and Fig. 13. A plot of the deformed shape of the panel predicted by the nonlinear analysis at an applied load of 240 kips is shown in Fig. 12(a). The deformed shape of the panel as measured with the VIC3D is shown in Fig. 12(b). 


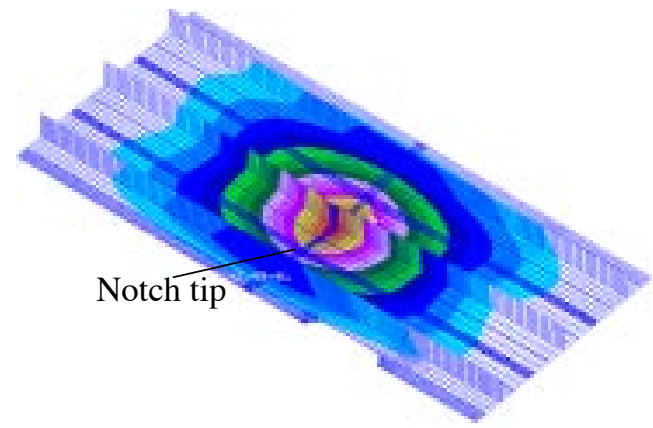

(a) Results obtained from nonlinear finite element analysis

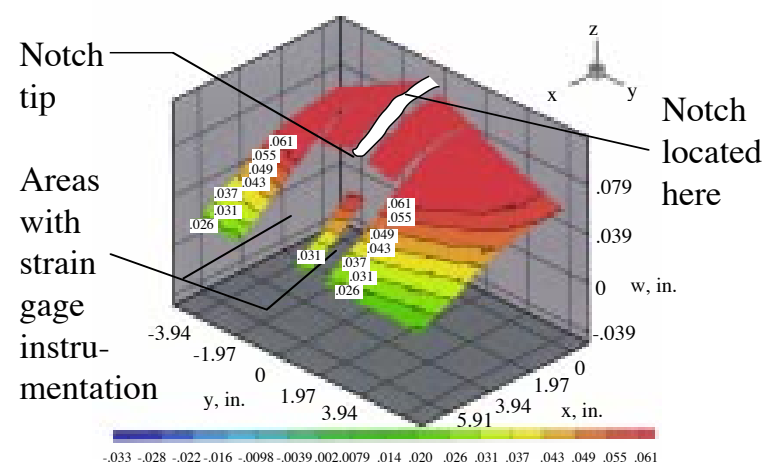

(b) Results obtained using the full-field displacement measuring system

Fig. 12 Deformed shape of the composite upper cover panel for an applied load of 240 kips.

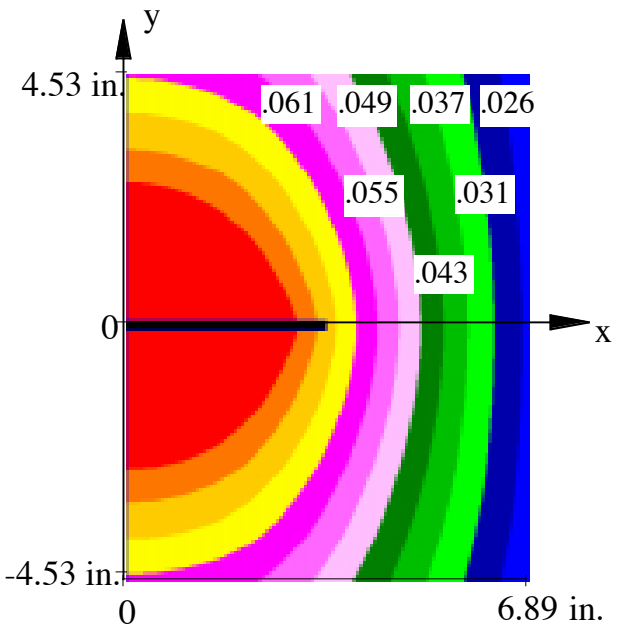

(a) Results obtained from nonlinear finite element analysis

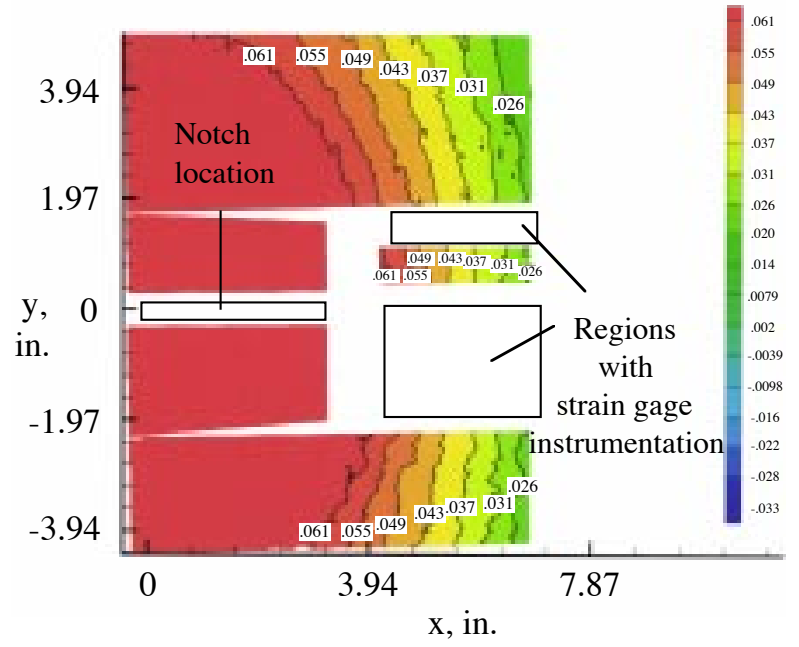

(b) Results obtained using the full-field displacement measuring system

Fig. 13. Out-of-plane displacement contours for the composite upper cover panel for an applied load of 240 kips.

Out-of-plane displacement contours obtained from the analysis at an applied load of 240 kips are shown in Fig. 13(a), and the corresponding contours obtained from the full-field measurements at this load are shown in Fig. 13(b). A comparison of the location and magnitude of the displacements suggest an excellent correlation between the two results.

A plot of the deformed shape of the panel as measured with the VIC3D system at an applied load of 300 kips is shown in Fig. 14.

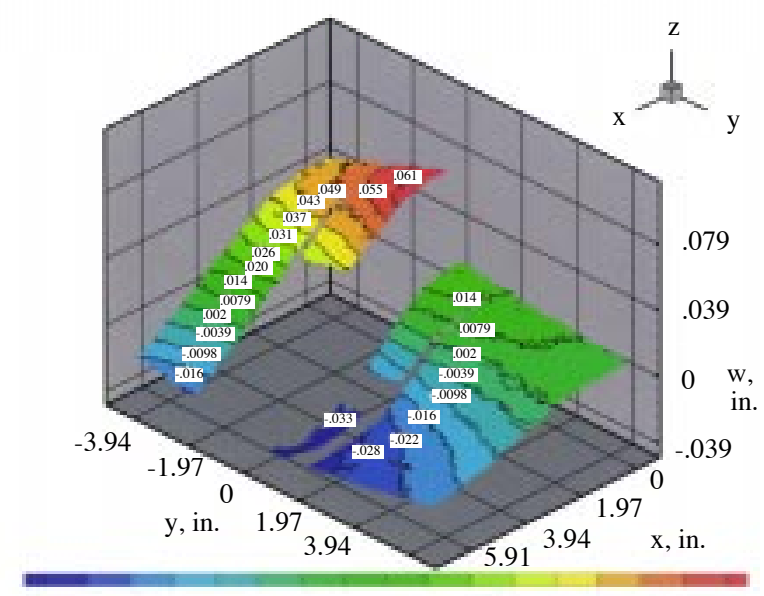

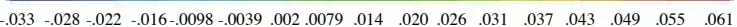

Fig. 14. Displacement contours for the deformed composite upper cover panel for an applied load of 300 kips (just prior to failure). 
This plot shows the reversal in the direction of the outof-plane displacement that occurred at loads greater than 225 kips, as indicated in Fig. 11.

\section{Lower Cover Panel Results}

Global-local full-field displacements were measured for the lower cover panel. The global displacements were measured over an area that was approximately 15in. wide by 27.5-in. long. The local area was centered on one of the notch tips, and it measured approximately 7.7-in. wide by 4.6-in. long. The full-field results were determined to be accurate for this test to within $\pm 5.9 \mathrm{x}$ $10^{-4}$ in. for the local results and to within $\pm 1.7 \times 10^{-3}$ in. for the global results. ${ }^{10}$

A comparison of measurements obtained from two DCDT's located on the stiffener side of the panel and full-field measurements obtained from the skin-side of the panel is shown in Fig. 15.

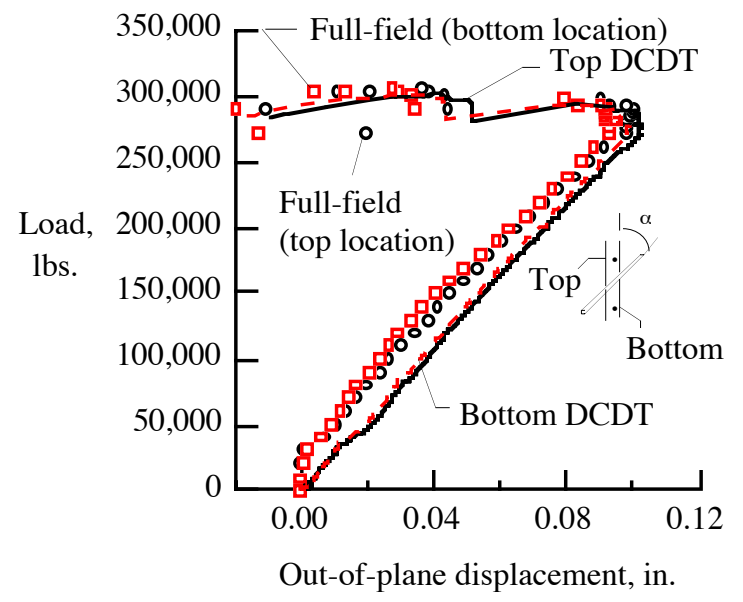

Fig. 15. Comparison of DCDT and full-field displacement results for the composite lower cover panel.

The DCDT's were located 1.83 inches above and below the horizontal centerline of the panel, and they were centered on the blade of the cut, center stringer. These DCDT locations are referred to in Fig. 15 as the top and bottom locations, respectively. The full-field results are compared to the point-wise DCDT results by displaying the displacements of the "speckle" that is most nearly opposite to that of the DCDT. The DCDT results are plotted in Fig. 15 as curves, and the full-field results are plotted as symbols. As shown in this figure, the agreement in the trends represented by the full-field results and the DCDT results is very good. The reversal in the direction of the out-of-plane displacement for loads greater than 280 kips was represented very well. This reversal in displacements resulted from propagating damage in the panel that initiated at the notch tip. Although the trends agree very well, there is a difference in the DCDT and full-field results. This difference grows to a maximum value of approximately 0.01 inches while the panel is being loaded to approximately 30 kips. The difference then remains relatively constant for loads less than approximately 280 kips. This difference is attributed to the difference in the location of the points being monitored, as discussed for the upper cover panel. Since the DCDT is actually in contact with the cut stringer, some localized inconsistencies in the measured displacements are possible as well.

Global full-field results obtained using the VIC3D system that show the deformed shape of the lower cover panel at applied loads of 240 kips and 290 kips (just prior to failure) are presented in Fig. 16.

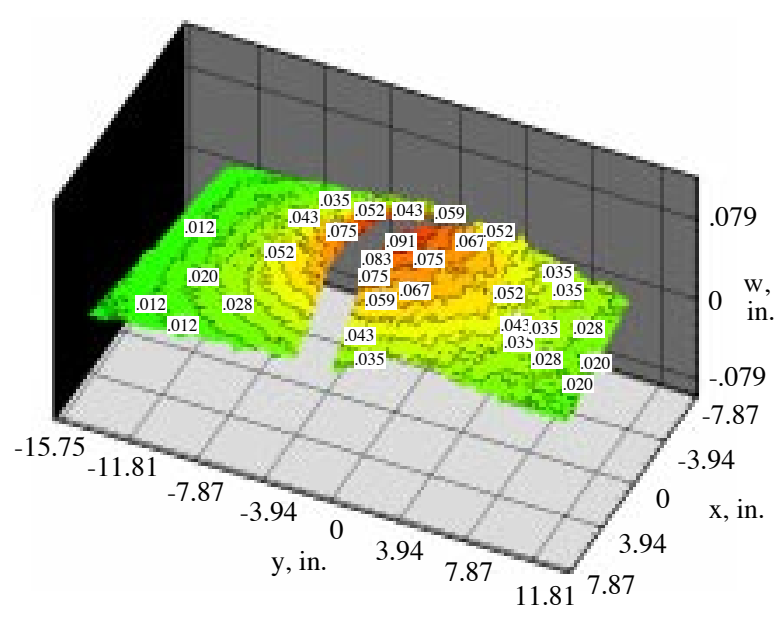

(a) Global full-field results for an applied load of 240 kips

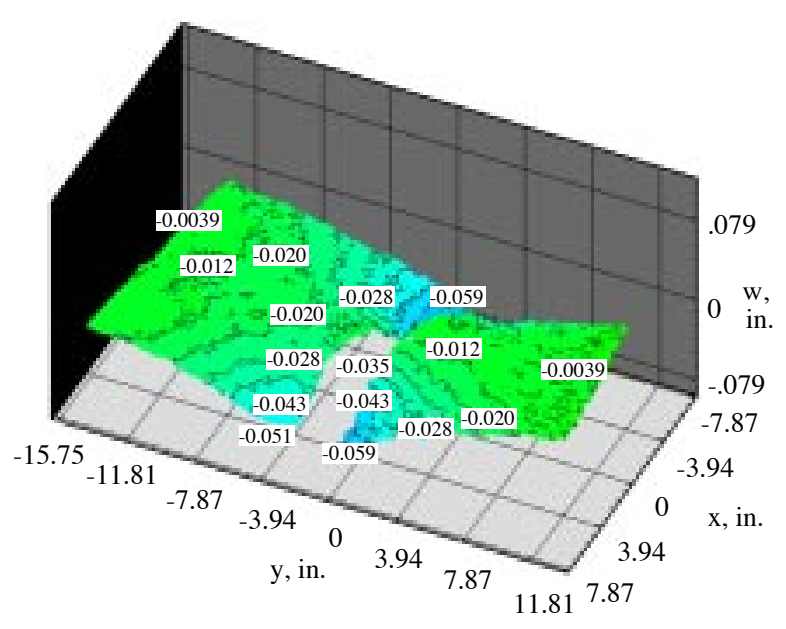

(b) Global full-field results for an applied load of 290 kips (just prior to failure)

Fig. 16. Displacement contours for the deformed composite lower cover panel for applied loads of 240 and 290 kips. 
The results shown in Fig. 16(a) suggest that the panel deformed outward in a symmetric manner. The results shown in Fig. 16(b) indicate that the panel deformed inward as it began to fail. These results are in agreement with the point-wise results presented in Fig. 15.

Out-of-plane displacement contours obtained using the VIC3D system are presented in Fig. 17 for an applied load of 290 kips (just prior to failure). The contour plots shown in this figure contain global and local full-field results that have been merged into one data set. There are no data shown in a selected area near the notch tip. This area was in the path of the growing notch, and the resulting outward brooming of the surface fibers caused very localized increases in the measured displacements that were not included in these results.

The results shown in Fig. 17(a) are for the entire global-local areas monitored during this test. A detailed view of the local results is shown in Fig. 17(b). One important result is the degree to which the global and local results can be matched at the boundaries between the two areas. Although the global results have less fidelity than the local results (evidenced by the contours shown in Fig. 17(b)), the two data sets match very well at the interface. Note also the small region of very large displacement gradients near the notch tip. These large displacement gradients are due to the presence of surface fiber failures that were present in the areas in which data were selected to be presented. These results indicate that a global-local approach to monitoring fullfield displacements can be useful for tracking the propagation of damage as a function of load as well as adding insight into the distribution of load in the structure as the damage propagates.

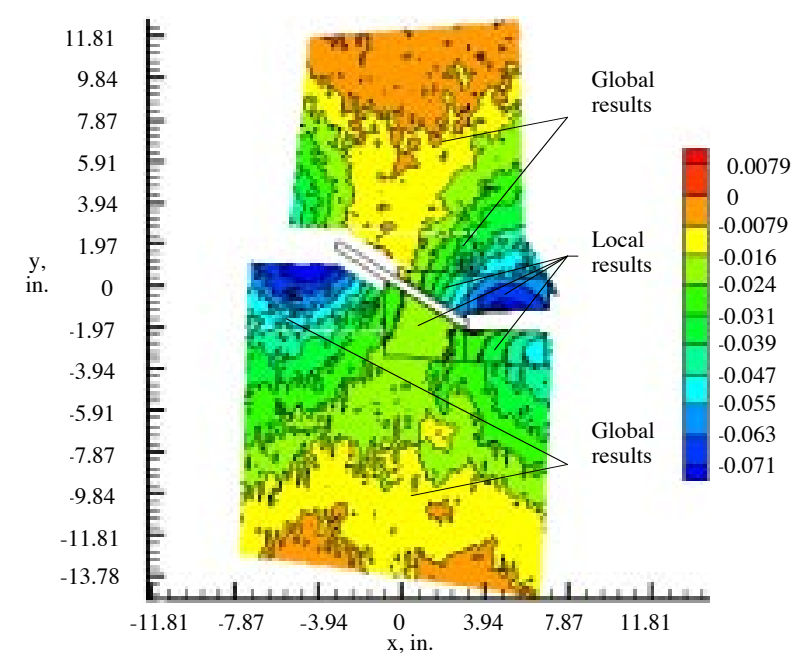

(a) Results for the complete field monitored

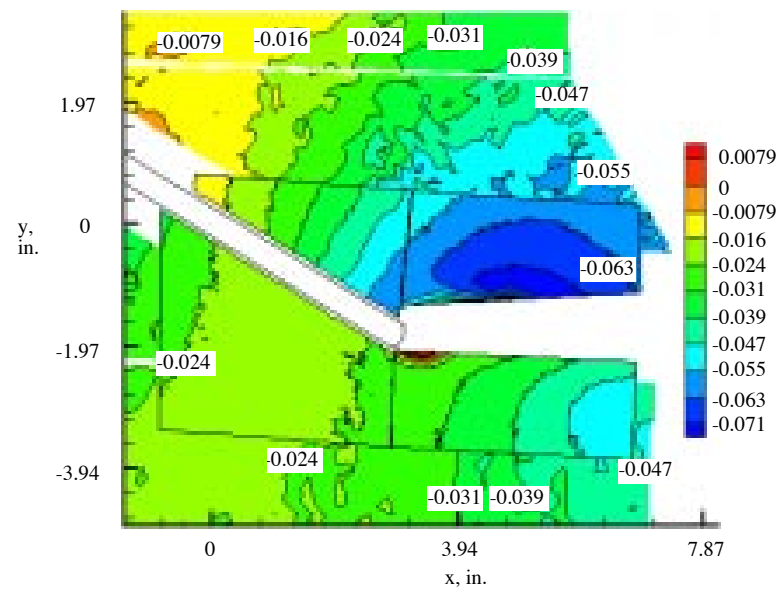

(b) Detailed view of the local results

Fig. 17. Full-field, global-local out-of-plane displacement contours measured with the VIC3D system for an applied load of 290 kips.

\section{Concluding Remarks}

The present paper describes an experimental and analytical evaluation of the compressive response of two graphite-epoxy, notched stiffened panels representative of primary composite wing structure. A threedimensional (full-field image correlation technique (VIC3D) was used to measure all three displacement components over global and local areas of the test panels. The accuracy of the displacements measured in the present study range from $\pm 3.5 \times 10^{-5}$ in. to $\pm 1.7 \times 10^{-3}$ in.

A simple tension test of a flat aluminum specimen with a centered, machined notch was used to calibrate the three-dimensional displacement measurement system with more conventional displacement measuring devices currently being used at the NASA Langley Research Center Structures and Materials Laboratory. The results of this test indicated excellent agreement between pointwise results obtained using the three-dimensional displacement measurement system and results obtained from a direct current displacement transducer and a fiberoptic displacement transducer.

Compression tests of two graphite-epoxy, notched stiffened panels were also conducted using the threedimensional displacement measurement system. Each of these three-stringer panels had a notch machined through the center stringer that was oriented at an angle to that stringer. Point-wise and full-field results obtained using the image correlation technique were compared to the experimental results and analytical results obtained using nonlinear finite element analysis. Results obtained using both global and global-local image 
correlation techniques were compared. The comparison of the image correlation results with the experimental and analytical results indicated excellent agreement of global and global-local results. The results obtained using the global-local displacement measurement technique also indicated that these types of measurements can be very important for obtaining a better understanding of the effect of local details like cutouts or damaged areas and propagating damage on the load distribution in built-up structures.

\section{References}

${ }^{1}$ Dally, J. M., and Riley, W. F., Experimental Stress Analysis, McGraw-Hill Book Company, 1978.

${ }^{2}$ Davidson, D. L., "Micromechanics Measurement Techniques for Fracture," Experimental Techniques in Fracture, edited by J. Epstein, VCH Publishers, 1993. ${ }^{3}$ Helm, J. D., McNeill, S. R., Sutton, M. A., "Improved Three-Dimensional Image Correlation For Surface Displacement Measurement," Optical Engineering, Vol. 35, No. 7, July 1996, pp. 1911-1920.

${ }^{4}$ Tsai, R. Y., "An Efficient and Accurate Camera Calibration Technique for 3D Machine Vision," Proceedings of the IEEE International Conference on Computer Vision and Pattern Recognition, 1986, pp. 364-374.

${ }^{5}$ Weng, J., Cohen, P., and Herniou, M., "Camera Calibration with Distortion Models and Accuracy Evaluation," IEEE Transactions of Pattern Analysis and Machine Intelligence, Vol. 14, No. 10, 1992, pp. 965-980.
${ }^{6}$ Luo, P. F., Chao, Y. J., and Sutton, M. A., "Application of Stereo Vision to 3-D Deformation Analyses in Fracture Experiments," Optical Engineering, Vol. 33, No. 3, 1994, pp. 981-990.

${ }^{7}$ Luo, P. F., Chao, Y. J., Sutton, M. A., and Peters, W. H., III, "Accurate Measurement of ThreeDimensional Deformations in Deformable and Rigid Bodies Using Computer Vision," Experimental Mechanics, Vol. 30 No. 2, 1993, pp. 123-132.

${ }^{8}$ Kahn-Jetter, Z. L., and Chu, T. C., "3-D Displacement Measurements Using Digital Image Correlation and Photogrammetry Techniques," Experimental Mechanics, Vol. 30, No. 1, 1990, pp. 10-16.

${ }^{9}$ Born, M., and Wolf, E., Principles of Optics, Pergamon Press, Third Edition, 1964, pp. 203- 233.

${ }^{10}$ Hanna, T. G., Three-Dimensional Deformation Measurements of Large Structural Panels, M.S. Thesis, University of South Carolina, 1998.

${ }^{11}$ Jegley, D. C., and Bush, H. G., Structural Test Documentation and Results for the McDonnell Douglas All-Composite Wing Stub Box, NASA TM 110204, April 1997.

${ }^{12}$ Markus, Alan, Thrash, Patrick, and Grossheim, Brian, "Manufacturing Development and Requirements for Stitched/RTM Wing Structure," Proceedings of the Fourth NASA/DoD Advanced Composites Technology Conference, NASA CP 3229, 1993, pp. 503-523.

${ }^{13}$ Brogan, F. A., Rankin, C. C., and Cabiness, H. D., "STAGS User Manual," Lockheed Palo Alto Research Laboratory Report LMSC P032594, 1994.

Table 1. Nominal elastic properties for the stitched/RFI material systems.

\begin{tabular}{lcc}
\hline \hline & AS4/3501-6 & AS4/IM7/3501-6 \\
\hline $\mathrm{E}_{\mathrm{x}}, \mathrm{Msi}$ & 8.17 & 9.98 \\
$\mathrm{E}_{\mathrm{y}}, \mathrm{Msi}$ & 4.46 & 4.45 \\
$\mathrm{G}_{\mathrm{xy}}, \mathrm{Msi}$ & 2.35 & 2.57 \\
$\mathrm{v}_{\mathrm{xv}}$ & .458 & .409 \\
\hline \hline
\end{tabular}

